\title{
METODOLOGÍA PARA LA CLASIFICACIÓN GEOMORFOLÓGICA DE LOS CURSOS FLUVIALES DE LA CUENCA DEL EBRO*
}

\author{
Elena Díaz Bea y Alfredo Ollero Ojeda \\ Dpto. de Geografía y Ordenación del Territorio, Facultad de Filosofía y Letras, \\ Universidad de Zaragoza. Pedro Cerbuna, 12, 50009 Zaragoza \\ aollero@unizar.es
}

\begin{abstract}
Resumen: Se establece una nueva clasificación de cursos fluviales a partir de variables geomorfológicas (pendiente del cauce, geomorfología del cauce-estilo fluvial- y geomorfología del valle) que ha sido aplicada a los sistemas fluviales de la cuenca del Ebro con el fin de obtener tramos fluviales funcionalmente homogéneos que sirvan como base para la aplicación de la Directiva Marco de Aguas $(2000 / 60 / \mathrm{CE})$. El resultado es la división de la red fluvial principal de la cuenca del Ebro en 1.436 tramos funcionales y la diferenciación de 78 tipos de curso fluvial, que pueden agruparse en básicos (32), de transición (25) y muy alterados (21). Por último, se sugiere una serie de propuestas generales para su futura ordenación, en especial para los tipos básicos de cauce, que representan el $83,5 \%$ del total de tramos.
\end{abstract}

Palabras clave: sistemas fluviales, geomorfología fluvial, Directiva 2000/60/CE, ordenación de cauces, cuenca del Ebro.

\begin{abstract}
A new classification of stream channels is established from geomorphological features (channel slope, channel pattern, valley morphology), which has been applied to the fluvial hydrosystems of the Ebro basin, in order to get some fluvial reaches, functionally homogenous, to be the base for the Water Framework Directive $(2000 / 60 / \mathrm{EC})$. As a result, the river network of the Ebro basin has been divided into 1.436 fluvial reaches, and 78 types of fluvial channels have been distinguished, which can be grouped into elementary (32), of transition (25) and very modified (21). Finally, some general proposals are suggested for its future management, particularly for the elementary types of stream channel, which represent the $83,5 \%$ of the total number of fluvial reaches.
\end{abstract}

Key words: fluvial hydrosystems, fluvial geomorphology, Water Framework Directive, river management, Ebro basin.

* Recibido: 19-01-05. Aceptado: 08-04-05. 


\section{Introducción}

Esta clasificación de cursos fluviales se ha diseñado para su aplicación a la red fluvial principal de la cuenca del Ebro, aportando una metodología sencilla que puede aplicarse a otras cuencas. Teniendo en cuenta las dimensiones del área de estudio (85.000 $\mathrm{km}^{2}$ ) y la importancia de la red fluvial principal $(12.000 \mathrm{~km})$ que ha sido objeto de la clasificación, ésta es más sencilla y directa que la aplicada en un trabajo anterior para Aragón (Ollero et al., 2003). En un primer momento se procedió a la aplicación de la clasificación de Rosgen (1994, 1996) de cauces y valles a nivel I (Sánchez Navarro et al., 2003), puesto que es la más completa y reconocida internacionalmente. Sin embargo, su puesta en práctica resultó dificultosa, ya que algunos de sus tipos no se ajustaban a los existentes en la cuenca del Ebro. Por este motivo, se ha terminado por establecer una nueva clasificación, en parte inspirada en la de Rosgen, pero muy fundamentada en la experiencia de los autores y adaptada a la complejidad de los sistemas fluviales de la cuenca del Ebro. También se ha tratado de ofrecer un producto sencillo y fácil de aplicar, de manera que el objetivo principal del presente trabajo es la detallada explicación de la clasificación para su posible posterior aplicación a otras áreas de estudio.

Con su aplicación en la cuenca del Ebro se ha logrado identificar de forma rápida y precisa los tramos fluviales interna y funcionalmente homogéneos, integrando tipos de cauce y valle a partir de mapas topográficos y ortofotos, sin recurrir al trabajo de campo. Así, se han localizado, cuantificado y caracterizado los tipos fluviales geomorfológicos básicos existentes en la cuenca del Ebro, así como los tipos de transición y alterados. De esta manera, la clasificación obtenida se convierte en base fundamental para:

- Organizar observaciones cualitativas y datos cuantitativos de diferentes elementos del sistema fluvial para la realización de estudios sobre su dinámica, añadiendo otros indicadores generales no incluidos en la clasificación (caudal, granulometría de los sedimentos, sustrato del lecho, vegetación de ribera, especies piscícolas, ambientes del corredor ribereño...), ya que van implícitos en la morfología del cauce resultante.

- Comparar distintos trabajos de diversos sistemas fluviales, partiendo de que las características de un determinado tipo de cauce se pueden extrapolar a otros tipos de ríos que están expuestos a procesos similares.

- Predecir la dinámica del río desde su morfología a partir de observaciones obteniclas en varios tipos de cauce.

- Valorar impactos potenciales (sensibilidad al impacto y potencial de recuperación) en los hidrosistemas fluviales de la cuenca.

Además, la clasificación geomorfológica de tramos fluviales homogéneos puede constituir el punto de partida para crear la red de seguimiento de la Directiva 2000/60/CE a escala de cuenca, estableciendo una red de puntos de muestreo para 
los indicadores hidromorfológicos, caracterizando las condiciones de referencia hidromorfológicas a partir de cambios de dinámica observados en fotografías aéreas antiguas, evaluando los indicadores de calidad hidromorfológicos, etc.

\section{Antecedentes en la tipificación hidromorfológica de ríos y riberas}

Todavía existe un vacío metodológico en la tipificación y en la caracterización hidromorfológica en la línea que establece la Directiva 2000/60/CE. Entre las recomendaciones publicadas hasta el momento destacan las del documento de la Commission of the European Communities (2002) Guidance standard CEN TC 230/WG 2/TG 5: N32 y las del grupo de trabajo 2.3 REFCOND (Wallin et al., 2002).

Sin embargo, hay numerosas clasificaciones de cursos fluviales desarrolladas desde criterios geomorfológicos, destacando por su relevancia y aplicación las de Leopold y Wolman (1957), Schumm (1963, 1977), Rust (1978), Kellerhals et al. (1972, 1976), Mosley (1987), Nanson \& Croke (1992), Downs (1995) o Montgomery \& Buffington (1993, 1997, 1998). Sin duda una de las clasificaciones más completas y aplicables a la ordenación es la de Rosgen (1994, 1996), que ha sido aplicada por Sánchez Navatro y Ollero (2003) al río Gállego.

En la mayoría de los países desarrollados han aparecido en la última década planes de ordenación, programas de conservación y restauración y normativas diversas que afectan a cauces y riberas, que han requerido establecer tipos, para posteriormente aplicar a cada tipo un modelo de gestión o de actuación. Entre las numerosas iniciativas científico-técnicas de clasificación y caracterización de cursos fluviales destacan la norteamericana "Rapid Stream Assessment Technique" (RSAT), las inglesals "River Habitat Survey" (RHS) (Raven et al., 1998), versión sencilla del "System for Evaluating Rivers for Conservation" (SERCON), o "Stream Reconnaissance" (Thorne, 1998), la francesa "SEQ-Physique" (Système d'Evaluation de la Qualité Physique des Cours d'Eau) (Tartar, 2001), la alemana Länderarbeitsgemeinschaft Wasser (LAWA-vorOrt) (LAWA, 2000) o la italiana "Indice de Funcionalitá Fluviale" (IFF) (Siligardi, coord, 2003). Una de las más interesantes es la iniciativa australiana del "River Styles Framework" (Brierley \& Fryirs, 2000; Fryirs \& Brierley, 2001; Brierley et al., 2002), herramienta con base geomorfológica que cuenta con el "Index of Stream Condition" (ISC) y que se integra en el programa AUSRIVAS (Australian River Assessment System) (Parsons et al., 2002). Cabe destacar también en esta línea por su notable valor metodológico la tesis doctoral de Schmitt (2001) aplicada a los ríos de Alsacia. Igualmente se ha trabajado en la clasificación de ríos de Sudáfrica (Heritage et al., 1997). En el manual de Kondolf y Piégay (2003) se dedica un interesante capítulo (Kondolf et al., 2003) a las clasificaciones geomórficas en el que se recogen algunas de las citadas y se hace referencia al especial desarrollo de esta línea de trabajo en los últimos años. 
Paralelamente a estas propuestas hay iniciativas que aportan criterios o métodos de valoración interesantes, como por ejemplo distintos índices de calidad de espacios ribereños: QBR (Qualitat del Bosc de Ribera) (Munné et al, 1998), IHF-GUADALMED (Bonada et al., 2002), RCE (Riparian, Channel and Environmental Inventory) (Petersen, 1992) o ISC (Index of Stream Condition) (Ladson \& White, 1999), que incluyen criterios hidromorfológicos.

En España no existen antecedentes en esta línea de trabajo, salvo la tipificación propuesta por Prat y Munné (1999) para la cuenca del Ebro la de Ollero et al. (2003, 2004) para la red fluvial aragonesa y la reciente propuesta de González del Tánago y García de Jalón (2004).

\section{Criterios de tipificación}

Se ha procedido a la división de la red fluvial principal de la cuenca en sectores funcionales homogéneos, es decir, tramos de río diferentes entre sí por su morfología (con un estilo geomorfológico propio) y que, por tanto, funcionan de forma distinta a los demás tramos, aunque interconectada (Amoros y Petts, eds, 1993). Para ello se han tenido en cuenta 3 criterios básicos: pendiente del cauce, geomorfología del valle y geomorfología del cauce (estilo fluvial). Cada tramo es internamente homogéneo en función de los tres criterios definidos, y cada cambio significativo en uno o más de ellos constituye el inicio de otro tramo diferente.

Como herramientas básicas se ha utilizado cartografía topográfica 1:50.000 y fotografías aéreas de la zona de estudio. En concreto, dada la gran amplitud de la cuenca del Ebro, se han consultado las ortofotos correspondientes al vuelo del SIG-Oleícola (1998) por encontrarse en formato digital y ser el vuelo más reciente y común a toda la zona estudiada. Éstas han sido especialmente útiles en la comprobación de la correcta identificación de los estilos fluviales (fundamentalmente en cursos trenzados y anastomosados), para apreciaciones sobre la sinuosidad de los cauces y para la detección de recientes infraestructuras y rectificaciones en los mismos. Como la división en tramos se ha elaborado a escala 1:50.000 se decidió que la longitud mínima de tramo fuera de $2 \mathrm{~km}$.

\subsection{Pendiente del cauce}

Este parámetro refleja fielmente el perfil longitudinal del río, que se adapta a los diversos desniveles que tiene que salvar en su recorrido mediante cambios en su secuencia longitudinal y creando a la vez las consiguiente formas del cauce: 
cascadas, saltos-pozas, rápidos, rápidos-remansos... Se ha dividido la red fluvial en tramos homogéneos de distinta pendiente con ayuda de un modelo digital del terreno (MDT 20 con retícula de $500 \mathrm{~m}$ ) con apoyo en los intervalos que establece Rosgen (1996) en su tipología. Este parámetro es útil para discriminar algunos tipos de cauce, sobre todo los muy pendientes $(>10 \%)$, y para separar los tipos de cauce de misma forma en planta (sinuoso, meandriforme, trenzado) en más o menos pendientes. Los intervalos escogidos son los que se representan en la figura 1.
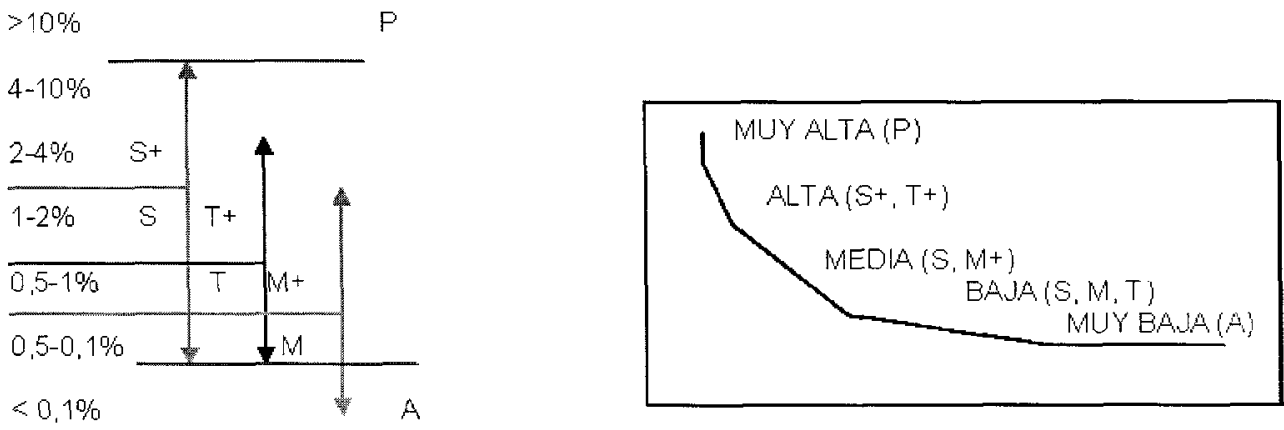

Figura 1. Intervalos de pendiente.

\subsection{Geomorfología del valle}

Se descartó la posibilidad de clasificar los valles por su origen, ya que generaba un excesivo número de tipos y no resultaba relevante en cuanto a la funcionalidad hidromorfológica en relación con los cauces. Así que se ha optado por una tipología más simple basada en la forma del relieve.

La forma del valle es un excelente indicador del grado de encajamiento del cauce en el valle y de su potencial desarrollo o progresión lateral, así como los controles estructurales a los que puede estar sometido. Asimismo, indirectamente aporta información cualitativa sobre elementos del sistema fluvial, como existencia de la llanura de inundación, extensión del corredor ribereño, presencia de terrazas, aporte directo de sedimentos desde las vertientes, etc. Los elementos clave de la forma del valle son la pendiente de las laderas y la anchura del fondo del valle. Así, pueden distinguirse 5 tipos de valle (figura 2).

La descripción de cada tipo de valle es cualitativa y no se han introducido en la clasificación valores cuantitativos sobre algunos de sus elementos -como la pen- 


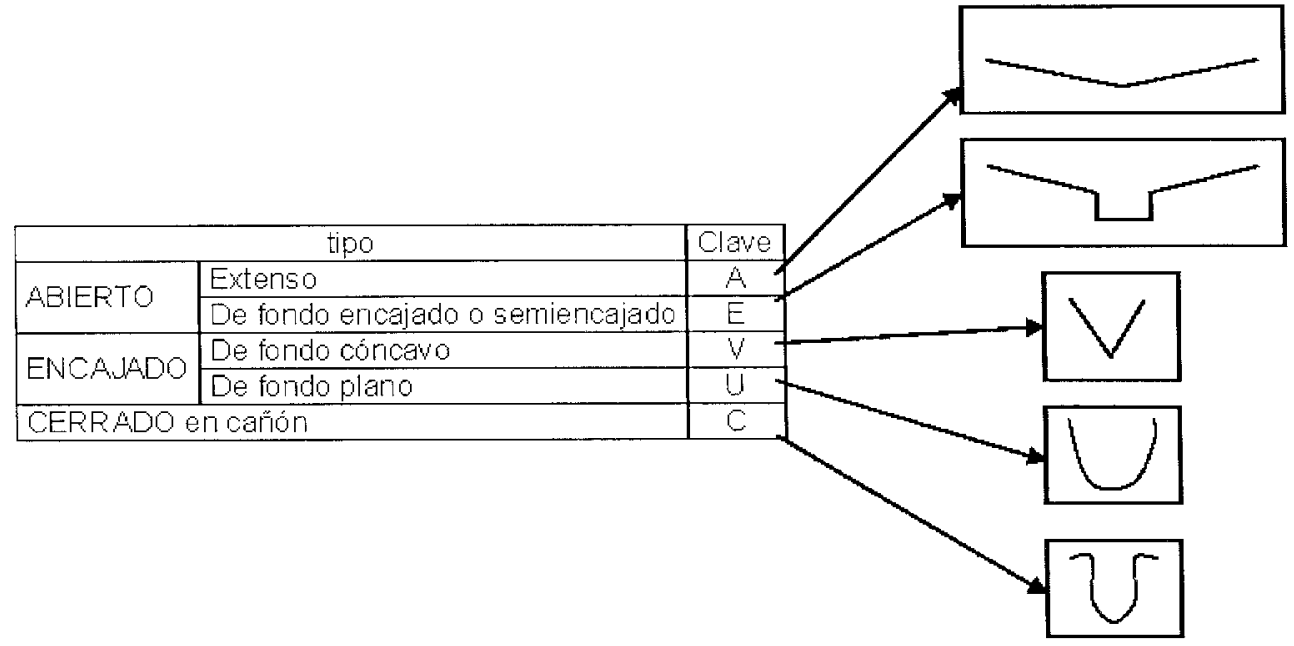

Figura 2. Tipos de valle.

diente de las vertientes o la anchura del fondo del valle- que podrían haber sido definitorios. Ello se debe a que el esfuerzo metodológico debe centrarse básicamente en los tipos de cauce, ya que son los que mejor definen el tipo geomorfológico final del tramo. Así, se considera que la descripción cualitativa de tipos de valle es suficientemente detallada y comprensible, teniendo en cuenta que en la presente clasificación se combinan tipos de cauce y valle, a diferencia de la de Rosgen (1996), que describe los tipos de valle pero no los aplica en la clasificación. Por ello se han agrupado y simplificado todo lo posible los tipos de valle en 5 , ya que de ser más habria resultado una canticlad inaceptable de tipos finales al combinarlos con los 10 tipos de cauce.

\subsubsection{Valle abierto extenso (A)}

Es un tipo de valle amplio, donde generalmente el desplazamiento lateral potencial del cauce en su fondo es bastante elevado (foto 1). Asociado a grandes depresiones, materiales deleznables, amplia llanura de inundación y presencia de terrazas, corresponde al tipo 8 de la clasificación de valles de Rosgen (1996). Es posible que la presencia de escarpes o de terrazas en una de las márgenes limite su desarrollo lateral, como por ejemplo el escarpe margo-yesífero que limita la margen izquierda de los tramos meandriformes libres del río Ebro entre Alagón y Zaragoza, y del río Aragón entre Caparroso y Marcilla. Pero la gran actividad lateral del cauce, constatada mediante fotografías aéteas antiguas, lleva a clasificar también esos casos como valles abiertos extensos. 


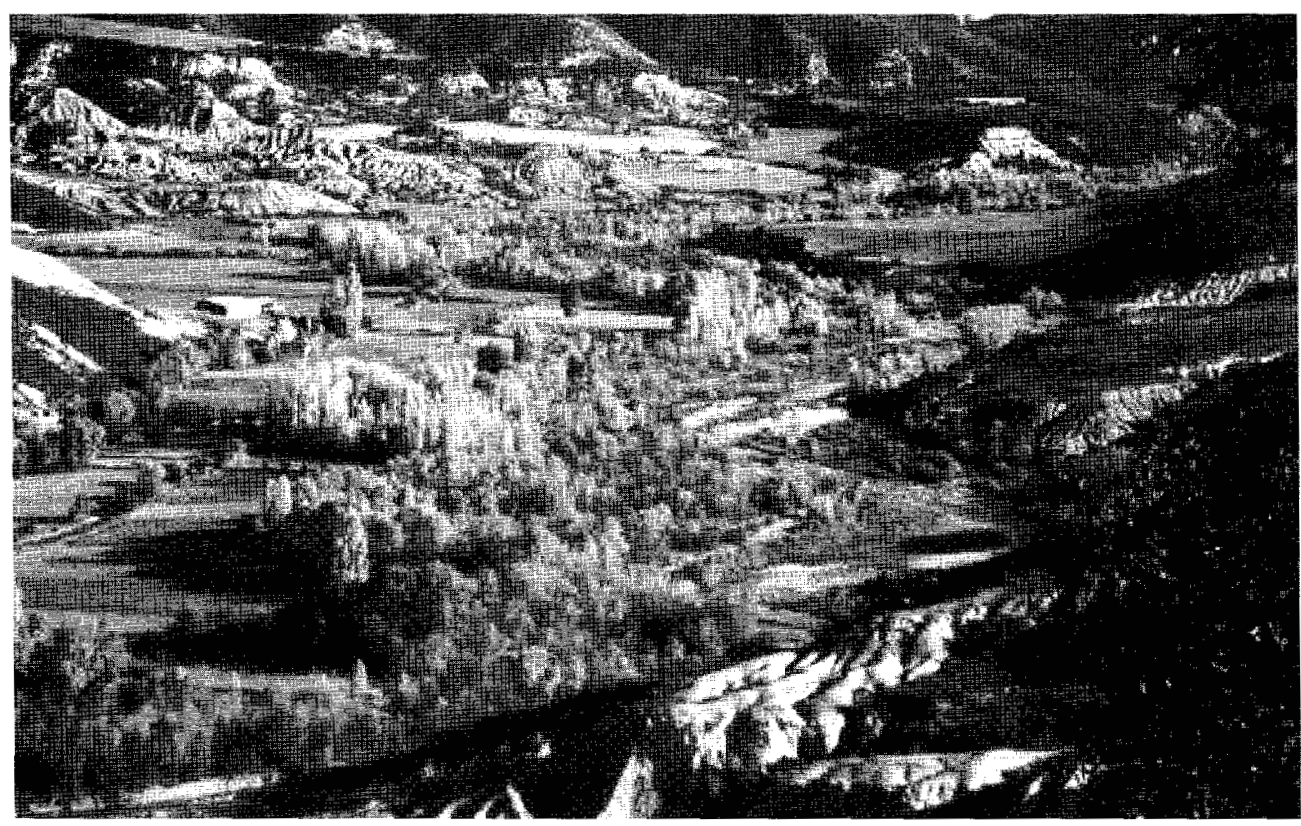

Foto 1. Valle de tipo A: río Veral en Berdún. Autora: E. Díaz Bea.

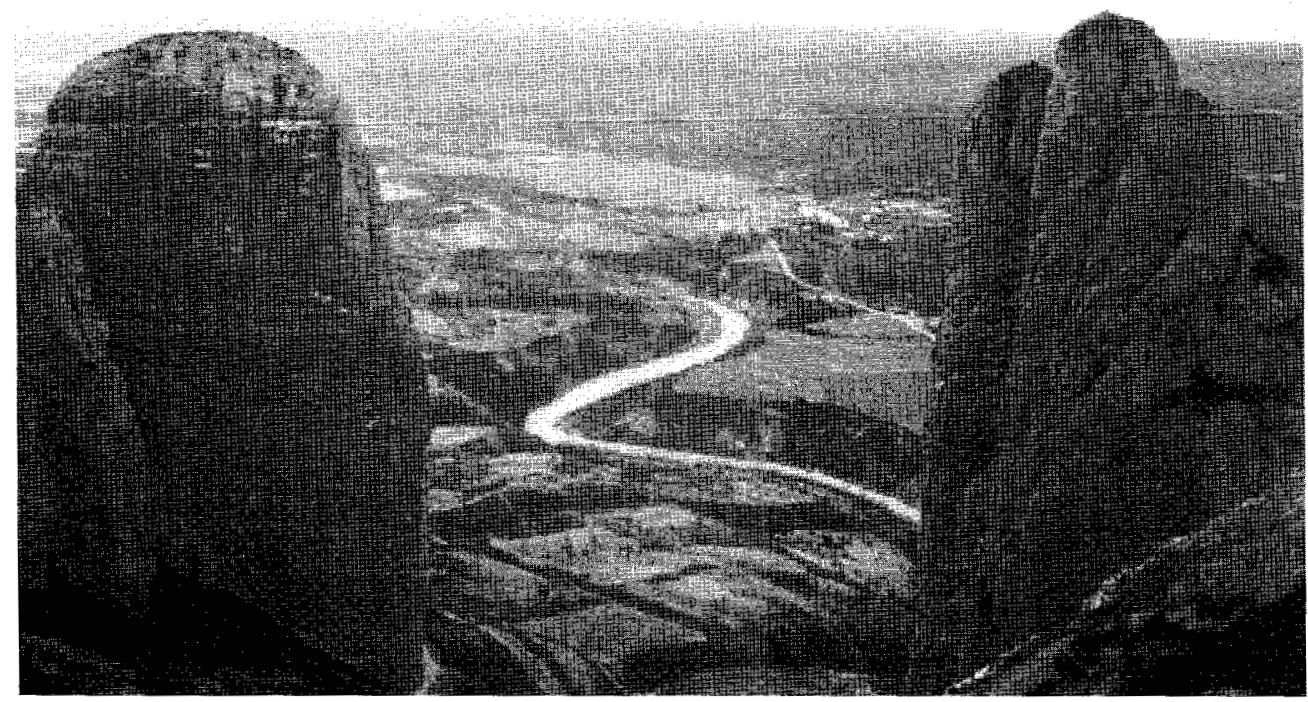

Foto 2. Valle de tipo E: río Gállego en Riglos. Autora: E. Díaz Bea. 


\subsubsection{Valle abierto de fondo encajado o semiencajado (E)}

El cauce está encajado o semiencajado de forma que ya no puede divagar lateralmente al máximo (foto 2). Suele encajarse en terrazas o glacis, por lo que su llanura de inundación es más reducida que en el caso anterior. Corresponde al tipo 4 de la clasificación de valles de Rosgen (1996).

\subsubsection{Valle encajado de fondo cóncavo (V)}

Son los típicos valles en $\mathrm{V}$ propios de áreas de montaña. En ellos el cauce queda constreñido por las dos laderas, que son de elevada pendiente (foto 3). Son característicos de cabeceras y gargantas, con abundantes procesos de vertiente y corredor ribereño estrecho, reducido a una hilera paralela al cauce. A veces quedan insertos dentro de un valle más amplio en U (por ejemplo, el tramo 3 del río Arazas, entre las Gradas de Soaso y el puente de Arripas). Corresponde a los tipos 1 y 2 de la clasificación de valles de Rosgen (1996).

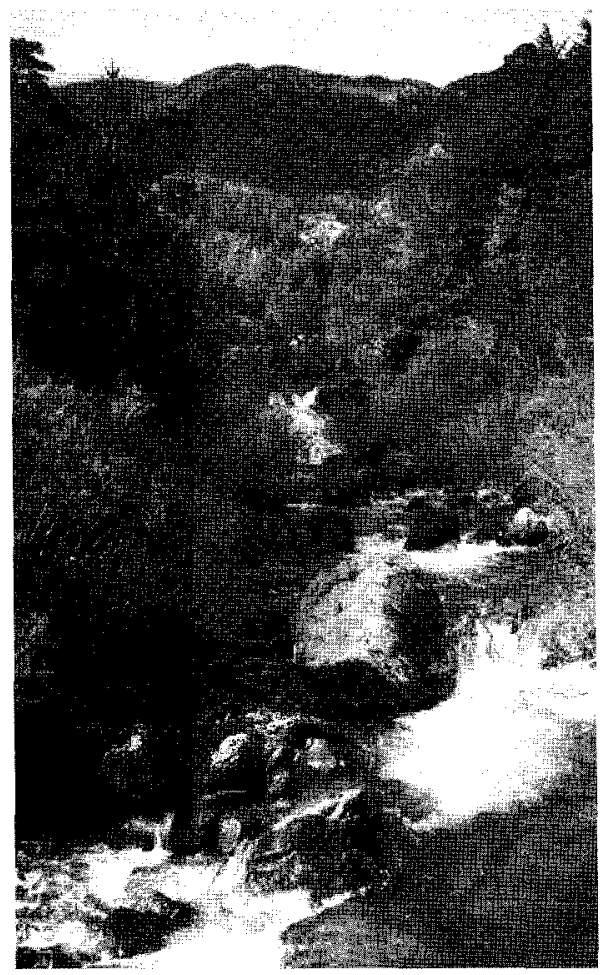

Foto 3. Valle de tipo V: río Estarrún. Autora: E. Díaz Bea. 


\subsubsection{Valle encajado de fondo plano $(U)$}

En él el cauce puede divagar lateralmente pero queda limitado por las paredes escarpadas del valle (foto 4). Dentro de este tipo se agrupan las artesas glaciares y los valles de fondo plano. Corresponde a los tipos 3 y 5 de la clasificación de valles de Rosgen (1996).

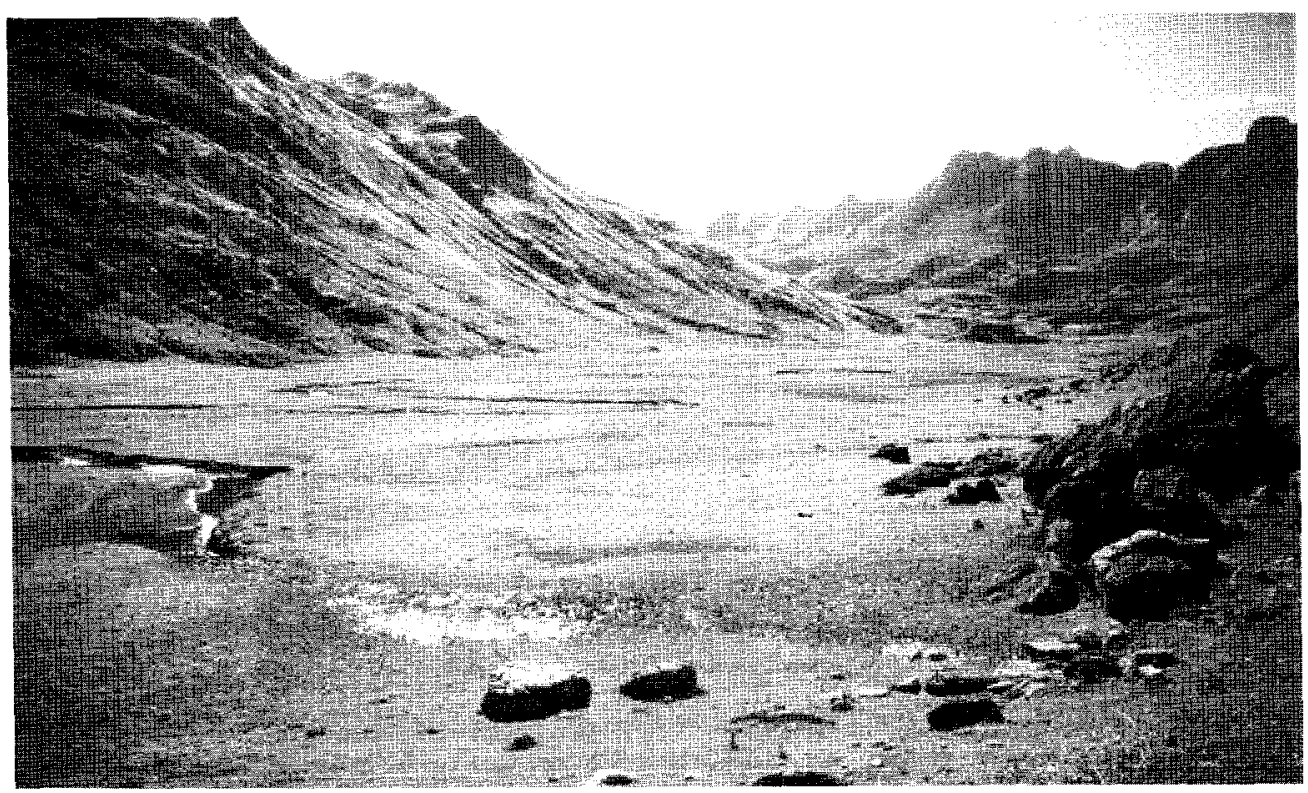

Foto 4. Valle de tipo U: río Subordán en Aguas Tuertas. Autora: E. Díaz Bea.

\subsubsection{Valle cerrado en cañón (C)}

Profundamente encajado con paredes casi verticales, generalmente en materiales calizos (cañones fluvio-kársticos), muy estrecho, carece de llano de inundación y su corredor ribereño es también muy reducido (foto 5). Corresponde al tipo 4 de la clasificación de valles de Rosgen (1996). También se incluyen en este grupo gargantas más abiertas por la alternancia de materiales resistentes y deleznables. Si sus vertientes son más inclinadas, en algunos casos se han considerado como valles en $\mathrm{V}$. 


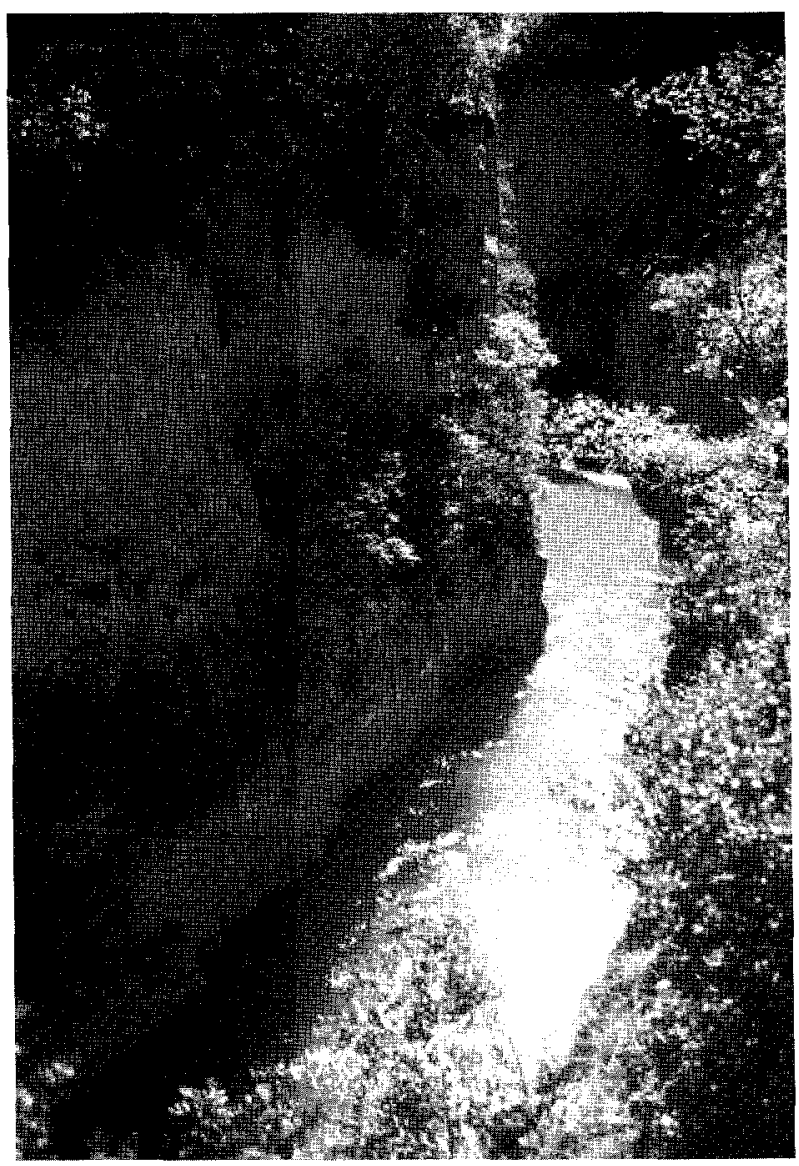

Foto 5. Valle de tipo C: río Veral en la Foz de Biniés. Autora: E. Díaz Bea.

\subsection{Geomorfología del cauce}

Es el criterio de tipificación más importante, ya que la forma del cauce (estilo fluvial) es el resultado de todos los elementos y procesos geomorfológicos que se dan en su cuenca. Para diferenciar la morfología en planta del cauce se ha atendido especialmente a si es único o múltiple y a su sinuosidad. La identificación entre cauces únicos (donde el índice de trenzamiento es $<1$ : rectos, sinuosos, meandriformes) y cauces múltiples (donde el índice de trenzamiento es $>1$ : trenzados y anastomosados) es muy clara, pero no ocurre así con los valores del índice de sinuosidad (Is). Los umbrales que separan los cauces rectos de los sinuosos y los cauces sinuosos de los meandriformes varían según distintos autores (tabla 1). 
Tabla 1. Umbrales entre tipos de cauce empleando el índice de sinuosidad, según diversos autores.

\begin{tabular}{|lcc|}
\hline & umbral recto-sinuoso & umbral sinuoso-meandriforme \\
\hline Leopold \& Wolman (1957) & 1,1 & 1,5 \\
Brice (1964) & 1,05 & 1,5 \\
Miall (1977) y Schumm (1977) & & $1,3(1,1$ si las sinuosidades son regulares) \\
Schumm (1981) & & 1,25 \\
\hline
\end{tabular}

Con base en el conocimiento previo de los autores sobre los cursos fluviales de la cuenca del Ebro, en la presente clasificación de cauces se ha tomado el umbral Is $=1,05$ para diferenciar cauces rectos de sinuosos y el umbral Is $=1,3$ para diferenciar cauces sinuosos de meandriformes. Como se aprecia en la tabla 1 son los umbrales más bajos, pero hay que tener en cuenta que en este estudio la longitud del cauce se ha calculado sobre el mapa topográfico en lugar de sobre la foto aérea, método que generalmente infravalora los valores del índice de sinuosidad.

Posteriormente se han combinado los valores de morfología en planta con los de pendiente, resultando 10 tipos básicos de cauce (figura 3).

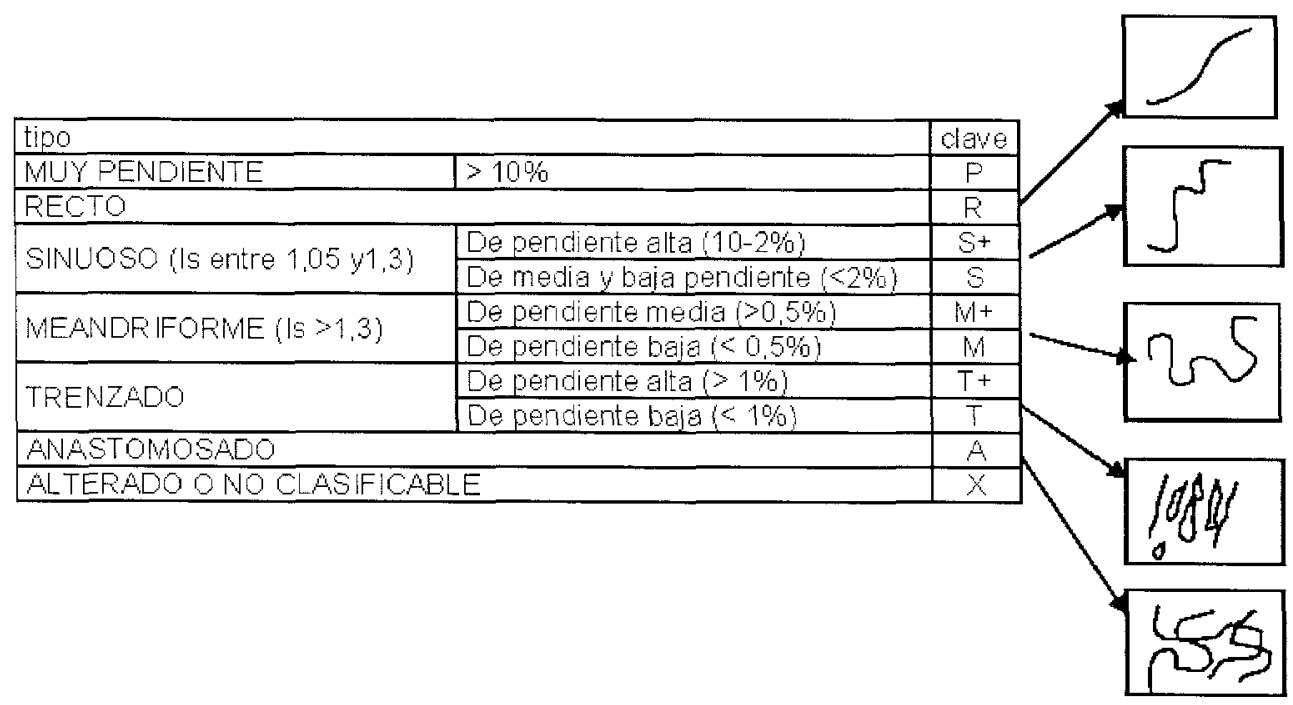

Figura 3. Tipos de cauce.

\subsubsection{Cauce muy pendiente (P)}

Típico de cabeceras muy pendientes, donde son frecuentes las cascadas y una estructura longitudinal de saltos y pozas (step-pool). Son muy encajados (baja ratio de 
anchura-profundidad) y tienen una gran capacidad de transporte e importante erosión lineal. Es el correspondiente al tipo Aa+ de la clasificación de cauces de Rosgen (1996).

\subsubsection{Cauce recto $\langle R\rangle$}

Exclusivamente reservado a cauces con forma en planta recta asociados a seguimiento de fracturas, estratos; es decir, a cauces sometidos a un control estructural. No tiene correspondencia con la tipología de cauces de Rosgen (1996).

\subsubsection{Cauce sinuoso de pendiente alta $(\mathrm{S}+)$}

Estructura longitudinal de saltos y pozas (step-pool) y rápidos, con pendientes todavía elevadas a moderadas y muy baja sinuosidad. Se puede emparejar con los tipos A, Ba, B ó G de la clasificación de cauces de Rosgen (1996).

\subsubsection{Cauce sinuoso de media y baja pendiente $(S)$}

Con estructura longitudinal de rápidos y remansos, comienza a describir algunas sinuosidades, pudiendo presentar barras laterales ocasionales. Corresponde a los tipos Bc y Gc de la clasificación de cauces de Rosgen (1996).

\subsubsection{Cauce meandriforme de pendiente media $(\mathrm{M}+)$}

Progresiva migración lateral del cauce y presencia de barras de meandro (pointbars). Estructura longitudinal de rápidos y remansos (riffle-pool) con mayor importancia de los rápidos. No tiene correspondencia con la tipología de cauces de Rosgen (1996).

\subsubsection{Cauce meandriforme de pendiente baja (M)}

Estructura longitudinal de rápidos y remansos (riffle-pool) con mayor importancia y longitud de los remansos. Mayor ratio de anchura de la banda de ameandramiento que en el tipo anterior. Consta de un amplio corredor y llanura de inundación, con posible presencia de cauces abandonados o galachos. Los point-bars están más desarrollados superficialmente. Es evidente una gradación granulométrica de los materiales, más grandes en el centro del cauce y más finos en las orillas. Corresponde a los tipos $\mathrm{Cb}, \mathrm{C}, \mathrm{Cc}$ (meandriforme), $\mathrm{Fb}, \mathrm{F}$ (meandriforme encajado) o $\mathrm{Eb}, \mathrm{E}$ (meandriforme profundizado, meandriforme tortuoso) de la clasificación de cauces de Rosgen (1996). 


\subsubsection{Cauce trenzado de pendiente alta $(T+)$}

Elevada carga de material sólido que obliga al cauce a dividirse y a depositar barras e isletas que son inestables o móviles en cada crecida. Estos múltiples cauces están englobados en un cauce mayor prácticamente rectilíneo con orillas escarpadas y erosivas, a veces muy deleznables, y generalmente en valles extensos. El perfil transversal del cauce es ancho y poco profundo, es decir, la relación anchura-profundidad es muy alta. Corresponde al tipo Db y D de la clasificación de cauces de Rosgen (1996).

\subsubsection{Cauce trenzado de pendiente baja $(T)$}

De una granulometría más variada que en el caso anterior, ya que pueden aparecer materiales finos, pueden presentar también zonas más estables. El corredor fluvial es más ancho y con más sinuosidades. A los lados de la banda activa o lecho mayor puede haber paleocauces colonizados por la vegetación que a veces se reactivan con el desplazamiento lateral de la banda activa. Corresponde al tipo Dc de la clasificación de cauces de Rosgen (1996).

\subsubsection{Cauce anastomosado (A)}

Estilo de muy baja pendiente, consta de múltiples y tortuosos cauces que son estables, separados por islas de material fino (arena, limos) tapizadas de vegetación herbácea. Además, hay brazos ciegos y zonas encharcadas. En cada cauce hay una estructura longitudinal de rápidos muy suaves y remansos (riffle-pool). Llanura de inundación extensa, ocupando todo el fondo del valle. Es un modelo típico de lagos glaciares colmatados, en valles tipo U. Correspondiente al tipo DA de la clasificación de cauces de Rosgen (1996).

\subsubsection{Cauce alterado o no clasificable $(X)$}

Cauces que por acción antrópica han perdido su morfología inicial, de tal manera que la actual es totalmente artificial: embalses, canalizaciones de hormigón, rectificaciones, dragados... Desde el punto de vista geomorfológico serían considerados "masas de agua artificiales o muy modificadas", tal como distingue la Directiva 2000/60/CE. No tiene correspondencia con la tipología de cauces de Rosgen (1996).

\section{Tipificación}

Se ha aplicado la clasificación de cauces y valles expuesta en el apartado anterior a todos los sectores funcionales en los que ha sido dividida la red fluvial principal de 
la cuenca del Ebro, segmentación efectuada también con base en la clasificación, es decir, marcando los límites de tramo en puntos de cambio de acuerdo con los criterios descritos. Para la definición de tipos se coloca en primer lugar la clave del tipo de cauce y a continuación la del tipo de valle, ya que el cauce tiene una clara prioridad, tanto funcional en el sistema fluvial como de cara a los objetivos de la Directiva.

\subsection{Tipos básicos}

Combinando los 10 tipos de cauce $(\mathrm{P}, \mathrm{R}, \mathrm{S}, \mathrm{S}+, \mathrm{M}, \mathrm{M}+, \mathrm{T}, \mathrm{T}+, \mathrm{A}, \mathrm{X})$ con los 5 tipos de valle ( $\mathrm{A}, \mathrm{E}, \mathrm{V}, \mathrm{U}, \mathrm{C}$ ) resultarían 42 tipos básicos, como puede observarse en la tabla 2 , ya que los tipos $\mathrm{P}$ y $\mathrm{X}$ no se asocian a ningún tipo de valle (los tipos $\mathrm{P}$ se insertan en valles iniciales de cabecera, y en los tipos $\mathrm{X}$ el valle es irrelevante porque, dada su alteración morfológica, no se consideran como ríos). En la red fluvial de la cuenca del Ebro, sin embargo, hay combinaciones de cauce y valle que no se han observado (las que en la tabla 2 no están en negrita), de manera que el número de tipos básicos presentes es de 32 . Es poco probable que las combinaciones no representadas puedan darse en algún sistema fluvial. En la figura 4 se representan gráficamente los principales tipos de entre los básicos.

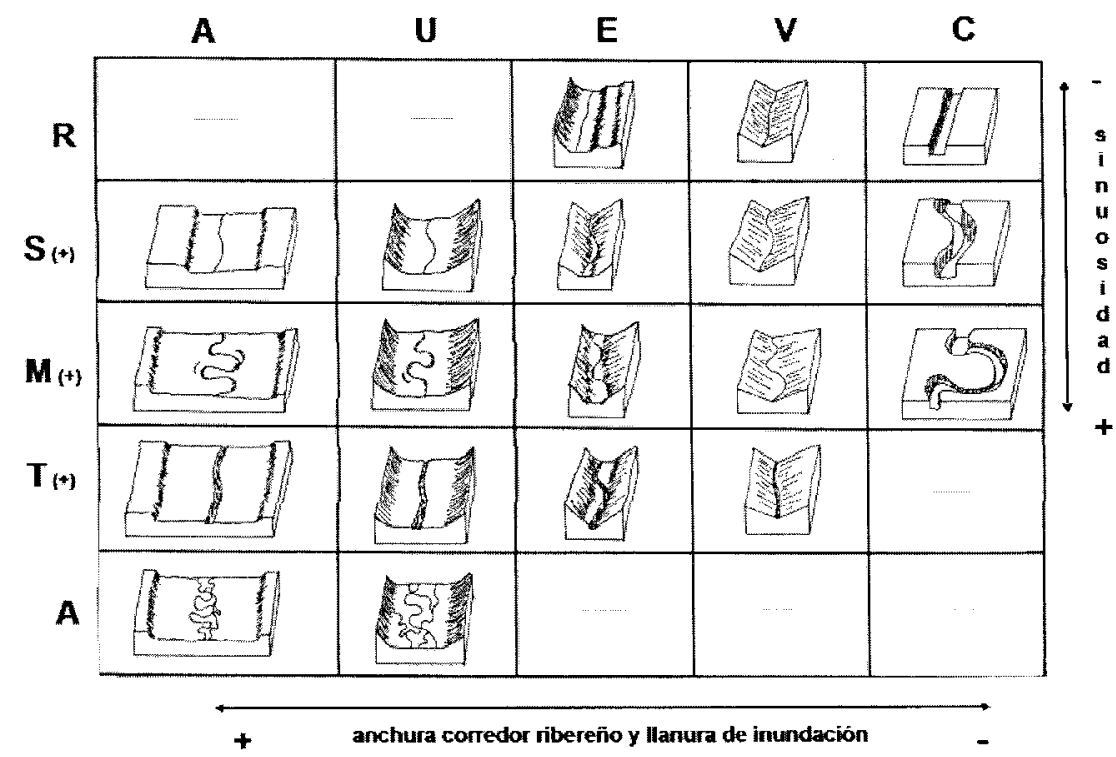

Figura 4. Tipos principales, resultado de la combinación de la forma del valle y del cauce y de acuerdo con los factores sinuosidad y anchura. 
Tabla 2. Los 78 tipos de cauce para la cuenca del Ebro.

\begin{tabular}{|c|c|c|c|c|c|}
\hline $\begin{array}{c}\widehat{d} \\
0 \\
0 \\
0 \\
0 \\
0 \\
0 \\
0\end{array}$ & $\begin{array}{c}\mathrm{RA} \\
\mathbf{S A} \\
\mathbf{S}+\mathbf{A} \\
\mathbf{M A} \\
\mathbf{M}+\mathrm{A} \\
\mathbf{T A} \\
\mathbf{T}+\mathbf{A} \\
\mathbf{A A}\end{array}$ & $\begin{array}{c}\mathbf{R E} \\
\mathrm{SE} \\
\mathbf{S}+\mathbf{E} \\
\mathbf{M E} \\
\mathbf{M}+\mathbf{E} \\
\mathbf{T E} \\
\mathbf{T}+\mathbf{E} \\
\mathrm{AE}\end{array}$ & $\begin{array}{c}\mathbf{P} \\
\mathbf{R V} \\
\mathbf{S V} \\
\mathbf{S}+\mathbf{V} \\
\mathbf{M V} \\
\mathbf{M}+\mathbf{V} \\
\mathrm{TV} \\
\mathbf{T}+\mathbf{V} \\
\mathrm{AV} \\
\mathbf{X}\end{array}$ & $\begin{array}{c}\mathrm{RU} \\
\mathbf{S U} \\
\mathbf{S}+\mathbf{U} \\
\mathbf{M U} \\
\mathbf{M}+\mathbf{U} \\
\mathbf{T U} \\
\mathrm{T}+\mathbf{U} \\
\mathrm{AU}\end{array}$ & $\begin{array}{c}\mathbf{R C} \\
\mathbf{S C} \\
\mathbf{S}+\mathbf{C} \\
\mathbf{M C} \\
\mathbf{M}+\mathbf{C} \\
\mathrm{TC} \\
\mathrm{T}+\mathrm{C} \\
\mathrm{AC}\end{array}$ \\
\hline 孞 & $\begin{array}{c}\text { StA } \\
\mathbf{S}+\mathbf{t A} \\
\mathrm{S}+\mathrm{aA} \\
\mathbf{M t A} \\
\mathbf{M}+\mathbf{t} \mathbf{A} \\
\mathbf{T s A} \\
\mathbf{T} \mathbf{A} \mathbf{A} \\
\mathbf{T}+\mathbf{s A}\end{array}$ & $\begin{array}{c}\text { StE } \\
\mathbf{S}+\mathrm{tE} \\
\mathrm{S}+\mathrm{aE} \\
\mathbf{M t E} \\
\mathbf{M}+\mathrm{tE} \\
\mathrm{TsE} \\
\mathrm{T} \mathbf{m E} \\
\mathrm{T}+\mathrm{sE} \\
\end{array}$ & $\begin{array}{c}\text { StV } \\
\mathbf{S}+\mathbf{t V} \\
\mathrm{S}+\mathrm{aV} \\
\mathbf{M t V} \\
\mathbf{M}+\mathbf{t V} \\
\mathrm{TsV} \\
\mathrm{TmV} \\
\mathrm{T}+\mathrm{sV}\end{array}$ & $\begin{array}{c}\text { StU } \\
\mathbf{S}+\mathbf{t U} \\
\mathbf{S}+\mathbf{a U} \\
\mathbf{M t U} \\
\mathbf{M}+\mathbf{t U} \\
\mathbf{T s U} \\
\mathbf{T m U} \\
\mathbf{T}+\mathbf{s U} \\
\end{array}$ & $\begin{array}{c}S t C \\
\mathbf{S}+\mathbf{t C} \\
\mathrm{S}+\mathrm{aC} \\
\mathrm{MtC} \\
\mathrm{M}+\mathrm{tC} \\
\mathrm{TsC} \\
\mathrm{TmC} \\
\mathrm{T}+\mathrm{sC}\end{array}$ \\
\hline 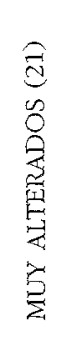 & $\begin{array}{c}\text { SxA } \\
\text { StxA } \\
\text { S+xA } \\
\text { S+txA } \\
\text { MxA } \\
\text { MtxA } \\
\text { XsA } \\
\text { TxA } \\
\text { TsxA } \\
T+x A \\
T+s x A\end{array}$ & $\begin{array}{c}\text { SxE } \\
\text { StxE } \\
\text { S+xE } \\
\text { S+txE } \\
\text { MxE } \\
\text { MtxE } \\
\text { XsE } \\
\text { TxE } \\
\text { TsxE } \\
\text { T+xE } \\
\text { T+sxE }\end{array}$ & $\begin{array}{c}\mathbf{S x V} \\
\text { StxV } \\
\mathbf{S}+\mathbf{x V} \\
\mathbf{S}+\mathbf{t x V} \\
\mathrm{MxV} \\
\mathrm{MtxV} \\
\mathrm{XsV} \\
\mathrm{TxV} \\
\mathrm{TsxV} \\
\mathrm{T}+\mathrm{xV} \\
\mathrm{T}+\mathrm{sxV}\end{array}$ & $\begin{array}{c}\text { SxU } \\
\text { StxU } \\
\mathbf{S}+\mathbf{x U} \\
\mathbf{S}+\mathbf{t x U} \\
\mathbf{M x U} \\
\text { MtxU } \\
\mathbf{X s U} \\
\mathbf{T x U} \\
\text { TsxU } \\
\mathbf{T}+\mathbf{x} U \\
\mathbf{T}+\mathbf{s x U}\end{array}$ & $\begin{array}{c}\text { SxC } \\
\text { StxC } \\
\text { S+xC } \\
\text { S+txC } \\
\text { MxC } \\
\text { MtxC } \\
\text { XsC } \\
\text { TxC } \\
\text { TsxC } \\
T+x C \\
T+s x C\end{array}$ \\
\hline
\end{tabular}

En la cuenca del Ebro más de la mitad de los tramos fluviales corresponden a tipos básicos sinuosos (56\%), siendo también de consideración la representación de cauces muy pendientes $(16,2 \%)$ y alterados $(12,5 \%)$. En menor medida se encuentran los demás tipos de cauce: meandriformes $(9,2 \%)$, trenzados $(5 \%)$, rectos $(1 \%)$ y anastomosados $(0,1 \%)$. La menor cuantía de cauces anastomosados debe explicarse por su rareza y pequeña longitud $(<2 \mathrm{~km}$.), que hace que en algunas ocasiones no lleguen a ser considerados como tramos diferenciados a esta escala de trabajo.

Dentro de los tramos sinuosos, se distingue entre los tipos de curso sinuoso de pendiente alta (los más abundantes son los ubicados en valles de tipo V) y los tipos de curso sinuoso de media y baja pendiente (más frecuentes en tipos de valles más abiertos, como los de tipo U y A). Entre los tramos meandriformes se diferencian los tipos de curso meandriforme de pendiente media (valles de tipo E y V) y los tipos de curso meandriforme de baja pendiente (tipos de valle E y A). En cuanto a los tramos trenzados, están representados los tipos de curso trenzado de pendiente alta (en tipos de valle $U$ ) y los tipos de curso trenzado de pendiente baja (en tipos de valle amplios $\mathrm{U}$ o A). Finalmente, los tipos de cauce recto abundan en tipos de valle $\mathrm{C}$. 


\subsection{Tipos de transición}

A los tipos básicos hay que añadir la posibilidad de tipos de transición que combinan dos estilos fluviales. En estas transiciones se coloca en primer lugar y en mayúsculas la letra del modelo más claro, aplicándose en ella la pendiente, y a continuación en minúscula la letra del modelo secundario. Por ejemplo, un tipo de transición meandriforme-trenzado con pendiente superior al $0,5 \%$ se clasifica como $\mathrm{M}+\mathrm{t}$.

Hay 8 tipos básicos de transición que, combinándolos con los tipos de valle, dan lugar a 40 tipos posibles, de los cuales en la cuenca del Ebro están representados 25 (en negrita en la tabla 2). Así pues, añadiendo a los 10 tipos básicos de cauce los transicionales resultan 18 tipos de cauce. Al combinarlos con los tipos de valle se suman a los 32 básicos otros 25 , dando lugar por tanto a 57 tipos.

Los ocho tipos básicos de transición son los siguientes:

- Sinuoso de pendiente alta y sinuoso de media y baja pendiente a trenzado $(\mathbf{S}+\mathbf{t}, \mathbf{S t})$ : son cauces sinuosos con gran carga de material que comienzan a presentar trenzamientos de 2 o más cauces en varios sectores. Ambos se dan en tipos de valle $\mathrm{U}$, aunque el de pendiente alta también aparece en valles encajados en $\mathrm{V}$.

- Sinuoso de pendiente alta a anastomosado ( $\mathrm{S}+\mathrm{a})$ : presenta varios cauces (pocos) pero son poco sinuosos, en tipo de valle U.

- Meandriforme de pendiente media a trenzado y meandriforme de pendiente baja a trenzado $(\mathbf{M}+\mathbf{t}, \mathbf{M t})$ : sería el equivalente al tipo meandriforme con barras (Ollero et al., 2003). Aunque ambos tipos son frecuentes en valles de tipo $\mathrm{E}$, el de pendiente media también se da en tipos de valle $\mathrm{U}$ y el de pendiente baja es abundante en tipos de valle $\mathrm{A}$.

- Trenzado de pendiente alta a sinuoso y trenzado de pendiente baja a sinuoso $(\mathbf{T}+\mathbf{s}, \mathbf{T s})$ : son antiguos cauces trenzados en los que, por escasez de aporte de sedimentos, se están incidiendo en un cauce único y más estrecho, dejando abandonados los extremos laterales del corredor que aparecen como terrazas subactuales. Ambos tipos se dan en valles de tipo $U$, aunque el de pendiente baja también aparece en valles abiertos de tipo A

- Trenzado de pendiente baja a meandriforme (Tm): es un cauce que ha sido trenzado pero que está cambiando su trazado a meandriforme con barras. Abundante en valles abiertos de tipo A, $\mathrm{U}$ y $\mathrm{E}$.

De todos ellos, los más abundantes son la transición de sinuosos a trenzados ( $\mathrm{St}$ : $53,4 \%$ y $\mathrm{S}+\mathrm{t}: 18,0 \%$ ) y la de meandriformes a trenzados (Mt: 8,3\% y $\mathrm{M}+\mathrm{t}: 7,5 \%$ ), es decir, la modificación de cursos sinuosos o meandriformes a cauces trenzados. En muchas ocasiones la transición se debe a la estacionalidad, apareciendo los trenzamientos en aguas bajas. 
En menor medida se encuentran transiciones de trenzado a sinuoso ( $\mathrm{T}+\mathrm{s}: 5,3 \%$ y Ts: $3,8 \%$ ) o a meandriforme (Tm: $2,2 \%$ ) y de sinuoso a anastomosado ( $\mathrm{S}+\mathrm{a}: 1,5 \%$ ). En casi todos estos casos se trata de procesos de deterioro del hidrosistema fluvial aguas abajo de embalses.

\subsection{Tipos con alto grado de alteración}

Se han considerado como tipos de cauce con alto grado de alteración a aquéllos en los que la modificación antrópica está muy presente en la mayor parte del tramo pero aún conservan varios sectores de dinámica natural (de lo contrario se clasificarían como X). Generalmente se aplica a sectores homogéneos donde se ha canalizado el cauce en varios tramos alternos.

De los tramos alterados en su mayor parte, los más abundantes están ubicados en valles amplios de tipo A y U (los más accesibles y ocupados antrópicamente) y son los $\mathrm{S}(51,5 \%$, ya que a su vez son los tipos de cauce más numerosos y los más fáciles de modificar por sus dimensiones) y los St (19,4\%, teniendo en cuenta que este tipo sería un cauce trenzado cuya forma en planta responde más a un cauce sinuoso debido a las numerosas canalizaciones).

\section{Resultados, conclusiones y perspectivas}

Se ha conseguido elaborar una tipología de cauces y valles aplicable a los sistemas fluviales de la cuenca del Ebro. En total se pueden diferenciar 78 tipos de curso fluvial que se pueden subdividir en básicos, de transición y muy alterados (tabla 2).

De esta manera no sólo se obtiene una caracterización de tipos básicos, sino que también se tiene en cuenta su cambio (ajuste de su morfología ante procesos distintos) y grado de alteración. Además, estos tipos pueden volver a reagruparse por tipos generales o por tipos de valle, dependiendo de los objetivos de ordenación que se persigan, lo que le confiere a esta clasificación versatilidad y una gran aplicabilidad. Dada su sencillez, esta clasificación puede ser aplicada y validada en otras zonas de estudio.

Dado que son los primeros resultados de una clasificación de cauces y valles recién aplicada, es necesaria una próxima fase de revisión y validación en campo de los tipos preasignados. Una vez superada dicha fase, esta clasificación podría ser la base para la elaboración de propuestas de ordenación de los espacios fluviales, por ejemplo en temas como la protección o conservación de determinados tipos geo- 
morfológicos singulares, el establecimiento del Dominio Público Hidráulico o de Espacios de Movilidad Fluvial adaptados a cada tipo geomorfológico, el establecimiento de una red de estaciones de referencia para variables hidromorfológicas, la restauración fluvial o el análisis de riesgos. En definitiva, se espera que sea una clasificación útil para la valoración de paisajes fluviales y para su ordenación o zonificación en función de su potencialidad.

A modo de muestra, se lanza una serie de propuestas generales de ordenación para cada grupo de tipos de cauce básico (tabla 3). No hay que olvidar que una clasificación de tipos de cauce supone una simplificación y una consiguiente agrupación de diferentes morfologías, por lo que para la ordenación de cada caso concreto habrán de estudiarse en profundidad múltiples aspectos (dinámica geomorfológica actual y pasada, grado de alteración en la misma, impactos principales,...) y tener en cuenta que la modificación de un tramo de cauce afecta también al resto.

Tabla 3. Valor geomorfológico a partir del grado de singularidad y dinámica y descripción de impactos y de propuestas de ordenación generales para cada grupo de tipos básicos de cauce.

\begin{tabular}{|c|c|c|c|c|c|}
\hline & $\begin{array}{c}\text { singularidad } \\
\text { cuenca }\end{array}$ & dinámica & $\begin{array}{l}\text { VALOR } \\
\text { geomorf. }\end{array}$ & impactos principales & $\begin{array}{l}\text { propuestas generales } \\
\text { de ordenación }\end{array}$ \\
\hline $\mathbf{P}$ & 4 & 4 & 4 & muy escasos & conservación \\
\hline $\mathbf{R}$ & 5 & 2 & 4 & muy escasos & conservación \\
\hline \multirow[t]{2}{*}{$\mathbf{S}$} & \multirow[t]{2}{*}{1} & \multirow[t]{2}{*}{3} & \multirow[t]{2}{*}{3} & $\begin{array}{l}\text { SA, SU rectificación, } \\
\text { canalización, } \\
\text { ahonclamiento del cauce }\end{array}$ & $\begin{array}{l}\text { restauración fluvial parál } \\
\text { mejorar el estado ecológico } \\
\text { conservación de los que están } \\
\text { en buen estado }\end{array}$ \\
\hline & & & & $\mathbf{S}+\mathbf{V}$ muy escasos & conservación \\
\hline \multirow[t]{3}{*}{$\mathbf{M}$} & \multirow[t]{3}{*}{5} & MA 5 & 5 & $\begin{array}{l}\text { escolleras } \\
\text { minicentrales } \\
\text { hidroeléctricas } \\
\text { motas } \\
\text { ocupación de la llanura } \\
\text { de inundación } \\
\text { dragados }\end{array}$ & $\begin{array}{l}\text { espacio de movilidad fluvial } \\
\text { especial protección }\end{array}$ \\
\hline & & MU 3 & 5 & escasos & especial protección \\
\hline & & MV, MC 5 & 4 & escasos & conservación \\
\hline $\mathbf{T}$ & 5 & ¿? & 5 & $\begin{array}{l}\text { dragados } \\
\text { extracciones de áridos } \\
\text { escolleras en orillas } \\
\text { presas de retención de } \\
\text { sedimentos } \\
\text { canalización en un } \\
\text { cauce menor }\end{array}$ & $\begin{array}{l}\text { espacio de movilidad fluvial } \\
\text { especial protección }\end{array}$ \\
\hline $\mathbf{A}$ & 5 & 1 & 5 & drenaje y canalización & especial protección \\
\hline $\mathbf{X}$ & 4 & 0 & 0 & $\begin{array}{l}\text { embalses } \\
\text { canalizaciones } \\
\text { cragados }\end{array}$ & $\begin{array}{l}\text { restringir su proliferación } \\
\text { restauración fluvial }\end{array}$ \\
\hline
\end{tabular}


Los cursos de tipo $\mathbf{P}$ son relativamente singulares en la cuenca del Ebro (representan un 16,2\% entre los tipos básicos), y su dinámica es activa. En general no sostienen impactos significativos dada su inaccesibilidad, por lo que se propone la conservación de este tipo de cauce. Aparte de su alto valor geomorfológico, es reseñable su alto valor escénico.

Los cursos de tipo $\mathbf{R}$ son muy singulares (1\%), aunque su dinámica es baja al tratarse de cauces encajados. Al igual que el anterior tipo no presentan impactos relevantes, por lo que se propone su conservación. También destacan sus altos valores geomorfológico y escénico.

El tipo S es el más común en la cuenca del Ebro (56\%) y su dinámica y valor geomorfológico pueden considerarse como medios. Los cauces sinuosos en valles abiertos como los $\mathrm{A}$ o los U son los que más impactos reciben, sufriendo cambios en su morfología mediante rectificaciones, ahondamientos del cauce y canalizaciones. Es debido generalmente al uso agrícola y urbano de los fondos de valle que son ajustados hasta la misma orilla del cauce. Por ello, serían convenientes programas de restauración fluvial para recuperar su morfología anterior y mejorar su estado ecológico. Aquéllos que conservan una morfología natural deben ser conservados mediante la protección de su corredor. Otro tipo de cauces sinuosos pero en valles encajados como S+V, SV, SE soportan menos impactos; éstos suelen ir ligados al paso de infraestructuras lineales que aprovechan estos estrechos valles (en los dos primeros casos) y al uso agrícola en la estrecha llanura de inundación (en el tercer caso). El tipo $\mathrm{S}+\mathrm{U}$ responde tanto a cursos de cabecera en valles glaciares pirenaicos como a cabeceras en valles abiertos del Sistema Ibérico; en general no están alterados pero se propone su conservación. Los tipos sinuosos en valles encajados en cañón deben conservarse por su singularidad dentro de los cauces sinuosos y por su valor escénico y biológico.

El tipo M es escaso en la cuenca del Ebro (9,2\%). Los cursos meandriformes de valles amplios (A, U) presentan un valor geomorfológico muy alto, siendo los MA muy dinámicos, con un cauce muy móvil y posibilidad de cortas de meandros. Éstos son los que más impactos reciben, debido a que su alta movilidad lateral choca con los intereses antrópicos de aprovechamiento de sus recursos y de ocupación de su amplia llanura de inundación: escolleras en orillas cóncavas para la fijación de los meandros, motas en las orillas para evitar la inundación del corredor (ocupado con uso antrópico), dragados del cauce para evitar cambios de trazado inesperados, minicentrales hidroeléctricas que "cortocircuitan" largos tramos del cauce por donde el caudal circulante es mucho menor al natural... Por ello, se propone para este tipo de cauce la delimitación de su Espacio de Movilidad Fluvial, es decir, un espacio libre de impedimentos donde el cauce pueda migrar libremente y tener una dinámica natural. Sólo así se conseguirá mantener y recuperar este tipo de cauce interesantísimo y con un alto valor ecológico y didáctico. 
Los tipos de cauce meandriforme en valle en U tienen una dinámica media, pero no soportan impactos de entidad, por lo que se recomienda una especial protección dado su alto valor geomorfológico, regulando las actividades antrópicas que podrían afectar en su dinámica. Los tipos meandriformes de valles más cerrados, como los $\mathrm{V}$, E o $C$, son mucho menos dinámicos y apenas reciben impactos de relevancia, por lo que se propone su conservación.

El tipo $\mathbf{T}$ es bastante singular en la cuenca del Ebro (5\%). Son cursos altamente dinámicos y a eso se le añade la imprevisibilidad del cambio en su dinámica, lo que les confiere un valor geomorfológico muy alto. Al igual que los MA, presentan una alta movilidad lateral y están muy alterados: se ha constatado la presencia de dragados y remoción de sedimentos para conseguir la presencia de un sólo cauce, extracciones de áridos, escolleras para evitar su movilidad lateral, presas de retención de sedimentos para evitar la temprana colmatación de embalses (lo que provoca la incisión de un sólo cauce debido al elevado poder de erosión del caudal líquido limpio), canalización en un cauce menor sinuoso (dando lugar a los tipos Stx) para estabilizar el cauce mayor, diques en el cauce mayor para desviar la corriente a un cauce más estrecho y estabilizar ese espacio, etc. Así pues, es necesaria también la delimitación de su Espacio de Movilidad Fluvial, que en este tipo de cauces debería abarcar al menos todo el cauce mayor y el corredor tibereño incluyendo paleocauces y zonas de vegetación de ribera estabilizadas. La ventaja de este tipo de cauces es que, dada su alta dinámica, una vez eliminados los impactos a los que se ven sometidos presentan un potencial de recuperación muy alto. Existe un escaso número de cauces trenzados en valles más encajados como los $\mathrm{V}$ y E, pero dada su peor accesibilidad están menos alterados por lo que se propone su conservación, ya que además suponen los casos más singulares de tipos de cauce trenzado.

El tipo $\mathbf{A}$ es el más escaso de toda la cuenca $(0,1 \%)$ y dada su baja dinámica son muy vulnerables, por lo que se debe sugerir una especial conservación.

Los cursos de tipo $\mathbf{X}$ son los tramos más artificiales de toda la cuenca; todavía representan un bajo porcentaje respecto a su presencia en la cuenca $(12,5 \%)$ y hacia ellos van orientados 2 medidas principales: no aumentar la proliferación de estos tipos de tramos y proceder a su restauración o rehabilitación en la medida de lo posible para la mejora de su estado ecológico.

Todas estas propuestas van dirigidas a la futura ordenación de los tipos básicos de cauce, que representan el $83,5 \%$ de la totalidad de tramos (1.428) en que se ha dividido la red fluvial principal de la cuenca del Ebro. Asimismo, habrá de prestarse especial atención a la existencia de los tipos de cauce con alto grado de alteración porque, aunque sólo representan el 7,2\% del total, se conoce su condición de referencia para plantear en ellos programas de restauración fluvial y conseguir su buen estado ecológico. 
En los tipos de cauce de transición $(9,3 \%)$ se aplicarán las propuestas de ordenación correspondientes a los tipos básicos que engloban, y habrá que tener en cuenta sobre todo las transiciones que suponen un deterioro del sistema fluvial, como las de trenzado a sinuoso o a meandriforme, para establecer en ellos medidas de corrección de impactos en la medida de lo posible.

\section{Agradecimientos}

El presente estudio ha sido financiado por la Confederación Hidrográfica del Ebro en el contrato de investigación "Tramificación de la red fluvial de la cuenca del Ebro" (Universidad de Zaragoza-OTRI 2004/0353).

\section{Bibliografía}

Amoros, C. et Petts, G.E. (eds.) (1993): Hydrosystèmes fluviaux. Paris, Masson.

Bonada, N. et al. (2002): Intercalibración de la metodología GUADALMED. Selección de un protocolo de muestreo para la determinación del estado ecológico de los ríos mediterráneos. Limmetica, 21(3-4), p. 13-33.

Brice, J.C. (1964): Channel patterns and terraces of the Loup River in Nebraska. U.S.G.S., Prof. Paper 422D.

Brierley, G. \& Fryirs, K. (2000): River Styles, a geomorphic approach to catchment characterisation implications for river rehabilitation in Bega catchment, New South Wales, Australia. Environmental Management, 25(6), p. 661-679.

Brierley, G., Fryirs, K., Outhet, D. \& Massey, C. (2002): Application of the River Styles framework as a basis for river management in New South Wales, Australia. Applied Geography, 22, p. 91-122.
Commission of the European Communities (2002): A guidance standard for assessing the bydromorphological features of rivers. CEN TC $230 /$ WG $2 /$ TG 5 N32, p. 1-21.

Downs, P.W. (1995): River channel classification for channel management purposes. In Gurnell, A.M. \& Petts, G.E. (eds.) Changing riwer channels, Chichester, Wiley, p. 347-365.

Fryirs, K. \& Brierley, G. (2001): A geomorphic approach to the identification of river recovery potential. Physical Geography, 21(3), p. 244 277.

González de Tánago, M. y García de Jalón, D. (2004): Hierarchical classification of rivers: a proposed for eco-geomorphic characterization of spanish rivers within the European Water Frame Directive. In García de Jalón, D. y Vizcaíno, P. (eds.) Procs. 5th Int. Symp. on Ecohydraulics, I, p. 205-212, Madrid.

Heritage, G.L., Van Niekerk, A.W. \& Moon, B.P. (1997): A comprehensive hierarchical 
river classification system. Geoökoplus, 4, p. $75-84$.

Kellerhals, R., Church, M. \& Bray, D.I. (1976): Classification and analysis of river processes. Journal of the Hydraulics Division, 102 , p. $813-829$.

Kellerhals, R., Neill, C.R. \& Bray, D.I. (1972): Hydraulic and geomorpbic characteristics of rivers in Alberta. Research Council of Alberta. River Engineering and Surface Hydrology Report 72(1), p. 1-52.

Kondolf, G.M. \& Piégay, H. (eds.) (2003): Tools in Fluvial Geomorphology. Wiley, Chichester.

Kondolf, G.M., Montgomery, D.R., Piégay, H. \& Schmitt, L. (2003): Geomorphic classification of rivers and streams. In Kondolf, G.M. \& Piégay, H. (eds.) Tools in Fhuial Geomorpbology, Wiley, Chichester, p. 171-204.

Ladson, A.R. \& White, L.J. (1999): An Index of Stream Condition Reference Manual. Melbourne, Department of Natural Resources and Environment.

LAWA (2000): Gewässerstrukturgütebewertung in der Bundesrepublik Deutscbland, Verfabren für kleine und mittelgroe Fliegewässer. Berlin, Länderarbeitsgemeinschaft Wasser.

Leopold, L.B. \& Wolman, M.G. (1957): River channel pattern braided, meandering and straight. U.S. Geological Survey, prof. paper 282B, p. 39-85.

Miall, A.D. (1977): A review of the braidedriver depositional environment. Earth Scences. Review, 13, p. 1-62.

Montgomery, D.R. \& Buffington, J.M. (1993): Channel classification, prediction of chan- nel response, and assessment of channel conditions. Washington State Dept. of Natural Resources, Timber/Fish/NWildland Agreement.

Montgomery, D.R. \& Buffington, J.M. (1997): Channel reach morphology in mountain drainage basins. Geological Society of America Bulletin, 109, p. 596-611.

Montgomery, D.R. \& Buffington, J.M. (1998): Channel processes, classification, and response potential. In Naiman, R.J. \& Bilby, R.E. (eds.) River ecology and management, New York, SpringerVerlag, p. 13-42.

Mosley, M.P. (1987): The classification and characterization of rivers. In Richards, K.S. (ed.) River channels environmental processes, London, Blackwell, p. 295-320.

Munné, A., Solà, C. y Prat, N. (1998) QBR: un indice rápido para la evaluación de la calidad de los ecosistemas de ribera. Tecnología del agua, 175, p. 20-37.

Nanson, G.C. \& Croke, J.C. (1992): A genetic classification of floodplains. Geomorpho$\log y, 4$, p. 459-486.

Ollero, A., Echeverria, M.T., Sánchez Fabre, M., Auria, V., Ballarín, D. y Mora, D. (2003): Metodología para la tipificación hidromorfológica de los cursos fluviales de Aragón en aplicación de la Directiva Marco de Aguas (2000/60/CE). Geographicalia, 44, p. 7-25

Ollero, A., Ballarín, D., Díaz Bea, E., Echeverría, M.T., Montorio, R., Mora, D. y Sánchez Fabre, M. (2004): Tipificación de los rios de Aragón. Departamento de Medio Ambiente, Gobierno de Aragón (informe inédito). 
Parsons, M., Thoms, M. \& Norris, R. (2002): Australian river assessment system review of pbysical river assessment methods. A biological perspective. Monitoring River Health Initiative, Technical Report $\mathrm{n}^{\circ} 21$, Commonwealth of Australia and University of Canberra.

Petersen Jr., R.C. (1992): The RCE a Riparian, Channel, and Environmental Inventory for small streams in the agricultural landscape. Freshwater Biology, 27, p. 295-306.

Prat, N. y Munné, A. (1999): Regionalización de la cuenca del Ebro. Confederación Hidrográfica del Ebro.

Raven, P.J., Boon, P.J., Dawson, F.H. \& Ferguson, A.J.D. (1998): Towards an integrated aproach to classifying and evaluating rivers in UK. Aquatic Conservation. Marine and Freshwater Ecosystems, 8(4) p. 383-393.

Rosgen, D.L. (1994): A classification of natural rivers. Catena, 22(3), p. 169-199.

Rosgen, D.L. (1996): Applied river morphology. Pagosa, Wildland Hydrology.

Rust, B.R. (1978): A classification of alluvial channel systems. In Miall, A.D. (ed.) Fluvial Sedimentology, 5, p. 187-198, Canadian Society Petroleum Geologists.

Sánchez Navarro, J.A. y Ollero, A. (2003) Aplicación de la clasificación de Rosgen al rio Gállego. Confederación Hidrográfica del Ebro (informe no publicado, ubicado en la web).

Sánchez Navarro, J.A., Ollero, A. y Díaz Bea, E. (2003): Protocolo para la aplicación de la clasificación de Rosgen a los ríos de la cuenca del Ebro. Confederación Hiclrográfica del Ebro (informe no publicado, ubicado en la web).

Schmitt, L. (2001): Typologie bydro-géomonphologique fonctionelle des cours d'eau. Recherche méthodologique appliquée aux systènes fluviaux d'Alsace. Thèse de Doctorat, Université Louis Pasteur, Strasbourg.

Schumm, S.A. (1963): A tentative classification of alluvial river channels. U.S. Geological Survey, circular 477.

Schumm, S.A. (1977): The fluvial system. New York, Wiley.

Schumm, S.A. (1981): Evolution and response of the fluvial system, sedimentologic implications. Society of Economic Paleontologists and Mineralogists Special Publication, 31, p. 19-29.

Siligardi, M. (coord) (2003): I.F.F. Indice de funzionalità fluviale. Roma, Agencia Nazionale per la Protezione dell'Ambiente.

Tartar, P. (2001): Mise en oeuvre du système d'évaluation de la qualité pbysique des cours d'eau sur l'Andelle. Note de synthèse. DIREN Haute-Normandie.

Thorne, C.R. (1998): Stream reconnaissance bandbook geomorphological investigation and analysis of river chamnels. Chichester, wiley.

Wallin, M., Wiederholm, T. \& Johnson, R.K. (2002): Guidance on establishing reference conditions and ecological status class boundaries for inland surface waters. Water Framework Directive Common Implementation Strategy Working Group 2.3 REFCOND, CIS-WFD. 